\title{
Estudio histórico del doblete catán-catana en lengua española
}

\author{
RAFAEL FERNÁNDEZ MATA \\ Universidad de Coimbra
}

\section{INTRODUCCIÓN}

En la primera mitad del siglo XVIII (entre los años 1726 y 1739), la Real Academia Española publicó su primera obra lexicográfica, comúnmente conocida como Diccionario de autoridades. Este sobrenombre le viene dado porque, para la descripción de las 69.410 entradas repartidas en seis tomos, la Academia se basó en la norma culta sustentada en el uso de los mejores escritores ${ }^{1}$.

A pesar de que tener como referentes a grandes literatos enriqueciera la lengua española, la directriz seguida por la Academia constituyó también el germen para el nacimiento y consolidación de errores, como tendremos ocasión de demostrar en nuestra investigación ${ }^{2}$. Concretamente, nuestro análisis se centrará en el estudio y descripción de la procedencia del cognado catán-catana, presente en la última, la vigésimotercera, edición del Diccionario de la lengua española de la Real Academia Española (2014).

A fin de distribuir y analizar adecuadamente los datos, dividiremos nuestro análisis en dos grandes apartados. En primer lugar, la sección dedicada a la voz catana, que a su vez se escinde en dos subdivisiones, una relativa a los datos actuales recabados sobre la voz catana, donde analizaremos las cuestiones relativas al étimo, la forma gráfica y acentuación, el significado y el área referencial y las características morfosintácticas de la palabra, y otra de carácter histórico, en la que dataremos su primer registro escrito en lengua española. En el segundo apartado trataremos de resolver el origen de la dualidad catán-catana, contrastando nuestra hipótesis con los datos recabados en diferentes fuentes.

Para concluir, quisiéramos advertir que, a fin de aligerar el texto, emplearemos una serie de abreviaciones relativas a los nombres de los corpus del español y las obras lexicográficas utilizadas en nuestra investigación, y que se pueden ver en el anexo I. En cuanto al anexo II, se trata de una tabla donde incluimos una relación de obras lexicográficas españolas de carácter diacrónico, y la información sobre catán-catana en ellas reflejada, obtenidas a través del portal en línea del Nuevo tesoro lexicográfico de la

${ }^{1}$ Como leemos en el prólogo original (http://www.rae.es/recursos/diccionarios/diccionariosanteriores-1726-1996/diccionario-de-autoridades), la información procede de aquellos escritores que «han tratado la Lengua Española con la mayor propriedad y elegancia: conociéndose por ellos su buen juicio, claridad y proporción, con cuyas autoridades están afianzadas las voces».

2 Como afirma la Academia, «el Diccionario de autoridades es la base de las distintas ediciones del diccionario usual de la Real Academia Española, deudoras, por tanto, del trabajo de aquellos primeros académicos». Para la fuente bibliográfica, véase la nota anterior. 
lengua española ${ }^{3}$. Tabla explicativa que esclarecerá, sin lugar a dudas, las referencias del epígrafe número 3.

\section{CATANA}

\subsection{Situación en el español actual}

Según el diccionario japonés Daijirin ${ }^{4}$, la voz «catana» procede del étimo 刀, cuya representación fonológica queda como sigue: $/ \mathrm{katana} / \mathrm{o} / \mathrm{katana} /{ }^{5}$. Grosso modo, posee tres acepciones en lengua japonesa: «1) Instrumento cortante que se usa como arma; 2) (En oposición a la espada ancha) Pequeño instrumento cortante utilizado con fines de autodefensa. Puñal; 3) Pequeño instrumento cortante. Cuchillo» ${ }^{6}$. De acuerdo con la información etimológica aportada por el Daijirin, su lectura y grafía actuales proceden de la suma de los sonidos de dos ideogramas: 片 /kata/, que significa 'uno de dos lados' y 刃 /na/, 'filo cortante'.

Después de examinar la evolución del significante catana en tres corpus lingüísticos del español ${ }^{7}$, observamos irregularidades gráficas en la historia de este japonesismo. Así, en el CORDE solo encontramos muestras de este vocablo con forma gráfica espanola: catana $(s)^{8}$. En el $C R E A$, sin embargo, se muestra una ligera preferencia por la grafía extranjerizante, katana(s): con 6 casos de katana y 2 de katanas, frente a los 5 de catana y 1 de catanas $^{9}$. Finalmente, en el CORPES XXI hallamos 13 casos de catana

${ }^{3}$ RAE (2016): Nuevo tesoro lexicográfico de la lengua española [en línea] <http://www.rae.es $>$ [Última consulta 27/05/2015].

${ }^{4}$ Información que hemos extraído del portal Kotobank (www.kotobank.jp), cuya naturaleza se aproxima, permítasenos la comparación, al NTLLE (RAE), ya que ofrece las acepciones recogidas en diferentes diccionarios japoneses modernos. Gracias a esta herramienta podremos conocer cuáles son las definiciones actuales de catana en japonés o si en esta lengua existe un significante similar a nuestro catán. En cuanto a 刀, visítese el siguiente enlace: https://kotobank.jp/word/\%E5\%88\%8044955\#E5.A4.A7.E8.BE.9E. 44955\#E5.A4.A7.E8.BE.9E.E6.9E.97.20.E7.AC.AC.E4.B8.89.E7.89.88.

5 Para la pronunciación de esta voz, hemos recurrido a la página virtual Forvo (http://es.forvo.com/), donde se recogen realizaciones articuladas de informantes nativos. Visítese el siguiente enlace para katana: http://es.forvo.com/word/katana/\#tr. Apréciese, asimismo, cómo hemos marcado en negrita los segmentos que, a nuestro parecer, se realizan con mayor fuerza espiratoria.

${ }^{6}$ Traducción y resumen de la descripción del Daijirin: «1) 武器して用いる邓物 Instrumento cortante que se usa como arma. (囚(両刃(もろは)の「剣( つるぎ)」に対して) 細し㭊の刃物 (En oposición a la espada de doble filo) Instrumento cortante largo y delgado con un solo lado afilado. (1)(短い茄差( わきざし)」に対して) 長い刃物 (En oposición a la espada corta) Instrumento cortante largo. 2) （長いた刃(たち）」に対して）小形護身用の刃物 腰刀(こしがなな）。短刀。 (En oposición a la espada ancha) Pequeño instrumento cortante utilizado con fines de autodefensa. Puñal. 3) 小せい刃物。 小刃(だたな)。Pequeño instrumento cortante. Cuchillo». Consúltese nota 4 para el enlace bibliográfico.

${ }^{7}$ Para el español pretérito usamos el CORDE y, para el actual, el CREA y el CORPES XXI. Advertimos que no incluiremos los textos que hemos encontrado nada más que cuando sea necesario; tampoco tendremos en consideración los resultados que no estén relacionados con los significados de catán-catana.

${ }^{8}$ Buscamos las siguientes formas sin resultados: [singular] «catán», «katán», «catan»y «katan» / «katana»; [plural] «catanes», «katanes», «catanos» $\mathrm{y}$ «katanos» / «katanas». RAE: Banco de datos (CORDE) [en línea]. Corpus diacrónico del español $<\mathrm{http}: / / \mathrm{www} . r a e . e s>$ [Última consulta 27/05/2015].

9 Buscamos las siguientes formas sin resultados: [singular] «catán», «katán», «catan» y «katan»; [plural] «catanes», «katanes», «catanos» y «katanos». RAE: Banco de datos (CREA) [en línea]. Corpus de referencia del español actual $<\mathrm{http}: / / \mathrm{www}$. rae.es $>$ [27/05/2015] 
frente a 12 de katana, y 1 de catanas frente a 4 de katanas $^{10}$. Apréciese, pues, que en la actualidad presenta irregularidad.

Su situación en las obras lexicográficas españolas resulta menos inestable, ya que en todas se recoge bajo la transcripción española: catana $^{11}$. En las obras lexicográficas extranjeras, observamos que, para el inglés, el $O E D$ lo transcribe mediante $k$, esto es, katana. Así se recoge también como forma primera en DOVLI y Zingarelli (aunque en este último aparece también catana) para el italiano. En cuanto al portugués, el DHLP y el DPLP emplean solo catana. Resultan llamativas las formas catan y cattan del $M W C D$, cuya etimología se debe al español. Sobre su patrón acentual, en lengua española no hay lugar a dudas, la ausencia de acentuación gráfica de las muestras de los corpus y las descripciones fonéticas del GDUEA y DClave indican que nos encontramos ante una palabra paroxítona.

Por lo que respecta al significado del término, detectamos consenso en los corpus del español y las obras lexicográficas, tanto españolas como extranjeras: todos los significados derivan de la primera acepción de este vocablo en lengua japonesa, es decir, en todos se recoge la idea de 'instrumento cortante que se usa como arma'. Si bien, en los corpus del español, observamos ligeras diferencias: mientras que en el CORDE suele estar empleado junto a sustantivos referidos a las armas (escopetas, arcos, flechas, rodela, morir, lanzas, guerra, etc.), en CREA y CORPES XXI se utiliza bien con palabras cuya área referencial es similar al del CORDE (arma de fuego, arma blanca, crimen, asesino, etc.), o bien se identifica con conceptos de la estética tradicional japonesa (guerrero japonés, samurai, kimono, ninjas, harakiris, etc.).

En cuanto a su integración en el sistema morfológico del español, los corpus y los diccionarios españoles coinciden en describirlo como un sustantivo femenino. Así lo demuestran los ejemplos de los corpus, donde viene acompañado por elementos adyacentes femeninos: la, las, una. Con este género ha pasado al portugués (cfr. DHLP y $D P L P$ ) y al italiano (Zingarelli y DOVLI —en este último como femenino invariable-). Su uso con morfema de plural en /-s/ (catanas), con cuantiosas muestras, también es sólido. No observamos preferencia por patrones combinatorios, más allá del circunstancial asesino de la catana.

\subsection{Descripción histórica: datación}

Manejamos varias fechas para el primer registro escrito de esta voz. En primer lugar, el CORDE ofrece el año de 1622 para el plural, catanas. En segundo lugar, el $D C E C H$ data en 1609 la forma catana $^{12}$. Sin embargo, gracias a la obra de Gil (1991: 28-29), podemos adelantar su primera aparición escrita al año de 1588, cuando el prior

${ }^{10}$ Buscamos las siguientes formas sin resultados: [singular] «catán», «katán», «catan» y «katan»; [plural] «catanes», «katanes», «catanos» y «katanos». RAE: Banco de datos (CORPES XXI) [en línea]. Corpus del Español del Siglo XXI (CORPES) <http://www.rae.es $>$ [27/05/2015].

11 Recogen el significante catana las siguientes obras lexicográficas españolas: $D R A E-2001$, GDUEA, DUE, DClave, DRAE-2014. No ofrecen ningún tipo de descripción o significante: $D E A \mathrm{y}$ $D U E A E$.

${ }^{12}$ Procedente del libro Sucesos de las islas Filipinas, de Antonio de Morga, publicado en 1609 en México, de acuerdo con Frago (1997: 109). 
Manrique del monasterio de San Agustín de la ciudad de Macán dirigió a Felipe II el 1 de marzo de $1588^{13}$ una misiva en la que escribe: «La gente es muy linpia y pulida en su trage, muy belicosa y valiente y armada de todas armas y buenos arcabuzes, lanças, catanas, que cortan a un hombre de un golpe, arcos, flechas, murriones y pecho y espalda» (Gil, 1991: 29). Por tanto, aunque raro ${ }^{14}$, es de esperar que este japonesismo fuera usado en castellano décadas anteriores a 1588, especialmente fuera de España, entre los primeros españoles en pisar tierra japonesa. Según Frago (1997: 112), este japonesismo aparece recogido por primera vez en suelo español en un acta del cabildo sevillano, fechada el 27 de octubre de $1614^{15}$.

De las lenguas occidentales consultadas, el portugués es la primera en recoger dicho japonesismo, en 1582, según Dalgado (1919: 281). El inglés, de acuerdo con el $O E D$, data su primera aparición en 1613. Por último, en italiano se ofrece la fecha de 1973 para la forma actual de katana, con $k$, mientras que registra el primer uso de catana en 1636 (Zingarelli).

\section{POSIBLE EXPLICACIÓN DEL DOBLETE CATÁN-CATANA}

\subsection{La forma catán en corpus y obras lexicográficas españolas}

Llegados a este punto, es hora de resolver cuál ha sido el origen de la dualidad catán-catana en lengua española, que parece no haber tenido parangón en ninguna otra lengua analizada ${ }^{16}$. En primer lugar, es necesario advertir que no hemos hallado ninguna muestra de la forma masculina catán - y cualquiera de sus posibles variantes- en los corpus del español, sea histórico o actual ${ }^{17}$. Si bien, la situación del significante masculino, catán, en los corpus contrasta enormemente con la de las obras lexicográficas españolas, pues, desde el segundo tomo de 1729 del Diccionario de autoridades (letra C) hasta la vigésimotercera edición del DRAE (2014) se ha venido utilizando en los diccionarios con la acepción 'especie de alfanje de procedencia indiana u oriental (sobre todo china)'. Incluso, desde el diccionario de Alemany y Bolufer (1917) hasta la vigésimoprimera edición del DRAE (1992), se le ha incluido una explicación etimológica que remite al árabe. Desde Alemany y Bolufer (1917) hasta la décimoséptima edición del DRAE (1947) se indicaba que el significado de su étimo era el de 'sable',

${ }^{13}$ Si bien, los hechos que relata son del año 1584 .

${ }^{14}$ Frago (1997: 110 y 111) sostiene: «este vocablo no debió pasar de un uso bastante minoritario y de constituir una verdadera rareza el objeto en cuestión». Para esta apreciación se basa en el dicen de la descripción aportada por la obra lexicográfica de Terreros y Pando (1786: 380, 2, consultada a través del NTLLE): «dicen que es una especie de alfanje indiano».

${ }^{15}$ Información que obtiene de GIL (1991: 396), quien relata la historia de la embajada de japoneses que llegó a España en septiembre de 1614 bajo el mando del samuray Hasekura.

${ }^{16}$ Solo en inglés. En el $M W C D$ aparecen las formas catan y cattan (a las que atribuye etimología española), mientras que el $O E D$ recoge tanto katana como cattan. Trataremos este asunto en líneas posteriores.

${ }^{17}$ Solo hallamos muestras en el CORDE: en primer lugar, con patrón llano, catan, que cuenta con 324 casos en 146 documentos; en segundo lugar, como palabra aguda, catán, con 16 casos en 3 documentos. En cuanto a su plural, de forma paroxítona, catanes, encontramos 4 casos en 4 documentos. No hay muestras para patrón oxítono, catanés. Ninguno de los casos anteriores hace referencia a su uso como 'especie de alfanje' o 'catana'. RAE: Banco de datos (CORDE) [en línea]. Corpus diacrónico del espa$\tilde{n} o l<$ http://www.rae.es> [Última consulta 27/05/2015]. 
mientras que desde la décimoctava edición del $D R A E$ (1956) hasta la vigésimoprimera (1992) se describía como 'cortante'. Por fin, en la vigésimosegunda edición del DRAE (2001) se incluyó el verdadero origen etimológico: «(Del jap. katana, espada)». Por lo que respecta a su significado, desde el Diccionario de autoridades (1729) hasta el de Zerolo (1895) su acepción de 'especie de alfanje, indiano, oriental o chino' convive con la de 'infanzón' - tipo de hidalgo- ${ }^{18}$.

\subsection{La forma catana en corpus y obras lexicográficas españolas}

Ya describimos en el segundo epígrafe la situación de la forma femenina, catana, en los corpus del español. Así que, a continuación, nos detendremos a analizar su tratamiento por parte de las obras lexicográficas. La primera en recoger esta voz, con el valor semántico actual de 'espada', es el diccionario de John Stevens (1706). Sin embargo, a pesar de que en el Diccionario de autoridades (1729) y en el de Terreros (1786) se incluya esta forma femenina en la descripción del vocablo masculino, $\mathrm{ca}$ $\operatorname{tán}^{19}$, lo cierto es que como sustantivo femenino apartado de la forma masculina no aparecería hasta el suplemento de Domínguez (1853). Anteriormente a la obra de Domínguez (1853), observamos que el significante femenino, catana, poseía tres significaciones pertenecientes a otra forma homófona y homógrafa: 1) Nombre propio de mujer (procedente de Catalina), desde el suplemento de la Academia (1803) hasta Domínguez (1853); 2) Capital de Sicilia (también con el significante Catina), desde Domínguez (1853) hasta Zerolo (1895); 3) Especie de papagayo, solo en el suplemento de la Academia (1803). En la última versión del DRAE (2014), existen dos sustantivos femeninos de diversa procedencia. El primero, de origen japonés, contiene las siguientes acepciones:

1) «Especie de alfanje que usaban los indios y otros pueblos del Oriente». Presente desde el Diccionario de autoridades (1729) - en una reciente modificación la Academia ha cambiado «que usaban los indios y otros pueblos del Oriente» por «de origen japonés»;

2) «Sable, en especial el largo y viejo, que usaban los policías». Sustantivo despectivo usado en Chile ${ }^{20}$. Su primera referencia se la debemos a Zerolo (1895);

3) «Automóvil o aparato mecánico viejo y destartalado». Utilizado en Cuba. Presente con descriptor geográfico cubano, aunque con otra significación ${ }^{21}$, desde Alemany y Bolufer (1917).

\footnotetext{
${ }^{18}$ No obstante, en el primer Diccionario histórico de la Real Academia Española (1936) aparece de nuevo su significado de 'infanzón'.

${ }^{19}$ En el Diccionario de autoridades (1729) leemos: «que en el Japón le llaman Catána», mientras que en el de Terreros (1786) encontramos: «y mas comunmente catana».

${ }^{20} \mathrm{Si}$ bien, en Alemany y Bolufer se incluyó otra región, Argentina, al descriptor geográfico. Con su acepción de americanismo usado en Chile y Argentina se mantuvo hasta el manual de la Academia (1989).

${ }^{21}$ La Academia enmendó o actualizó la acepción cubana en la vigésimosegunda edición del $D R A E$ (2001), puesto que desde Alemany y Bolufer (1917) hasta la vigésimoprimera edición del DRAE (1992) se describía como «cosa pesada, tosca, deforme».
} 
Por otro lado, como sustantivo femenino procedente del nombre propio de mujer, Catalina, posee la siguiente acepción:

1) «Loro verde y azul. Hay otras variedades». El primero en ofrecer esta voz como americanismo, procedente de Venezuela, es Alemany y Bolufer (1917). Si bien, ya en el suplemento de la Academia (1803), leemos una descripción que tal vez pueda estar relacionada con el significado actual: «Especie de papagayo menor que los comunes, y que tiene media cabeza colorada».

\subsection{Hipótesis del origen del cognado}

Después de examinar minuciosamente la información obtenida en las diferentes obras lexicográficas españolas, analizar las muestras recogidas por los corpus del español y comprobar la inexistencia de artículos u otras publicaciones que arrojaran algo de luz sobre la dualidad entre catán y catana, estamos en disposición de sostener que todas las pruebas apuntan a que la forma masculina, catán, podría haber sido un error iniciado por el Diccionario de autoridades (1729) y promovido durante casi cuatro siglos por la Real Academia Española y otros lexicógrafos que tomaban esta institución como referencia.

Las pruebas que nos inclinan a mantener esta teoría son las siguientes:

1) En primer lugar, y fundamental, no hemos hallado ni un solo ejemplo de catán en los corpus del español, solo su perpetuación a lo largo de las diferentes obras lexicográficas estudiadas - vid. ANEXO II-.

2) El único caso de significante plural, catanes, lo encontramos en el Diccionario de autoridades (1729), que a su vez lo extrae de la Conquista de las islas Malucas de Bartolomé Leonardo de Argensola ${ }^{22}$, concretamente en el libro sexto, folio 200, en el que leemos: «los quales dieron sus muestras y reseñas con picas y catanes (son poco diuersas de alfanjes) con demostraciones de gente alegre y satisfecha». Apréciese que, para el Diccionario de autoridades, la Academia olvidó tener en cuenta la paráfrasis explicativa, en la que se incluye el adjetivo femenino diuersas, con el que claramente Argensola se refiere a catanes. Esto quiere decir que, para el autor, la palabra era, sin lugar a dudas, femenina. La Academia se basa en un solo ejemplo de Argensola, que además toma con género equivocado ${ }^{23}$, a pesar de que el autor jamás estuvo en los Reinos Orientales a los que su libro se refiere ${ }^{24}$. Incluso es muy probable que nunca hubiera visto una catana, aunque sí era conocedor, al menos, del género del japonesismo en cuestión.

${ }^{22}$ Fue impreso en Madrid el 4 de mayo de 1609, aunque en la Licencia y aprouacion del Ordinario, se indica que fue mandado a imprimir por Felipe III el 30 de diciembre de 1608 (Argensola 1609).

${ }^{23}$ En esta misma equivocación incurrieron los autores del $D C E C H$, puesto que, al indicar que la forma singular catán se registra por primera vez en Argensola, estaban infiriendo que catanes era el plural para catán.

${ }^{24}$ Eso inferimos al leer en el folio 1 de la Conquista: «Yo Escriuo la Reducción de las Islas Malucas, introduzido de nueuo por Don Pedro de Acuña, Gouernador de las Filipinas, y General de la armada Española [...]» (Argensola 1609: fol. 1). En el folio 2, continúa: «A mi pues, por librar de este peligro [el autor pretende evitar que la historia no se narre de manera fidedigna], sucesso tan importante como el de Ternate, cabeça de todo el Maluco, me la mandaron escriuir, a tiempo que biuen los que interuinieron en el con las manos, y con el consejo. Y estoy informado de lo que para esto es menester, tanto que espero suplir con la verdad la falta del ingenio» (Argensola 1609: fol. 2). Por tanto, parece ser que él nunca 
3) Por otro lado, el CORDE demuestra, con los numerosos ejemplos de su uso femenino, que este era el género con que la voz japonesa había sido adaptada a la lengua española, tanto en la Península, como fuera de ella. De acuerdo con los primeros testimonios escritos del contacto entre castellano-hispanos y japoneses, que Gil (1991) recoge y analiza en su obra, desde las primeras transcripciones siempre se utilizan formas femeninas, tanto en singular como en plural:

\begin{tabular}{|c|c|}
\hline FORMA & AÑO \\
\hline «[...] catanas, que cortan a un hombre de un golpe» (Gil 1991: 29). & 1588 \\
\hline $\begin{array}{l}\text { «[...] le envió una catana, que es espada de las de allá, estimada en un reino } \\
\text { por su fineza y adorno» (Gil 1991: 45). }\end{array}$ & 1592 \\
\hline «[...] no saven vivir sin probar sus catanas» (Gil 1991: 63). & 1595 \\
\hline «[...], catanas» (Gil 1991: 95). & 1609 \\
\hline «También me dio una espada que llaman catana [...]» (Gil 1991: 163). & 1610 \\
\hline «[...], $\operatorname{catanas}^{25} »($ Gil 1991: 170). & 1610 \\
\hline «[...] dos espadas ricas que llaman catanas $»$ (Gil 1991: 195). & 1610 \\
\hline «[...] y con dos catanas ceñidas [...]» (Gil 1991: 198). & 1610 \\
\hline $\begin{array}{l}\text { "Y el de sus espadas y catanas también es cosa rara, porque hay catana que se } \\
\text { aprecia en cien mil ducados» (Gil 1991: 202). }\end{array}$ & 1610 \\
\hline $\begin{array}{l}\text { «[...] y luego echa mano a la catana que traen ceñida y córtanse por medio } \\
{[\ldots] »(\text { Gil 1991: 207). }}\end{array}$ & 1610 \\
\hline «[...] lanças y espadas y dagas que llaman catanas [...]» (Gil 1991: 217). & 1610 \\
\hline$《[\ldots]$ una catana enastada $[\ldots] »($ Gil 1991: 355). & 1614 \\
\hline «[...], y que le dava su catana $[\ldots] »($ Gil 1991: 366$)$. & 1614 \\
\hline$\langle[\ldots]$, le ciñen la catana $[\ldots] »($ Gil 1991: 374$)$ & 1614 \\
\hline «[...] meter mano a una catana [...]» (Gil 1991: 374). & 1614 \\
\hline «[...] una daga que llamavan catanas [...]» (Gil 1991: 396). & 1614 \\
\hline «[...] con estas dos catanas $[\ldots] »($ Gil 1991: 397$)$ & 1614 \\
\hline «[...] les hurtaron una catana $[\ldots] »($ Gil 1991: 410). & 1614 \\
\hline «[...] sacó una catana, que es como un alfange [...]» (Gil 1991: 463). & 1617 \\
\hline «[...] la catana avía pegado un golpe [...]» (Gil 1991: 463). & 1617 \\
\hline
\end{tabular}

Además de la lengua española, en otras tan próximas como la portuguesa o la italiana, que también tuvieron representación en los Reinos Orientales ${ }^{26}$, el japonesismo se adaptó como voz femenina (cfr. DHLP, DPLP, DOVLI y Zingarelli). Por ejemplo, según el primer tomo del Glossário luso-asiático de Dalgado (1919: 281), el primer registro escrito en lengua portuguesa de la palabra catana tiene lugar en 1582, para el singular catanà .

estuvo presente en aquellas tierras, sino que fue informado de todo lo allí acaecido a fin de narrar por escrito, de la manera más fiel, los acontecimientos históricos.

${ }^{25}$ Resulta curiosa la adaptación femenina plural, catanes, de Argensola (1609), ya que para su Relación del Japón, Rodrigo de Vivero (1610), personaje que sí tuvo contacto directo con la cultura y el territorio japonés, emplea constantemente los significantes catana y catanas. Incluso hallamos a pocas líneas de catanas los plurales masculinos tatames y quimones (cf. Gil 1991: 170). ¿Pudo Argensola, tal vez por analogía con las formas masculinas, construir un plural femenino con terminación -es?

${ }^{26}$ Confróntense el mencionado Gil (1991) u otros como Cabezas (1994) o Cid Lucas et alii (2011). 
4) Por las vagas e imprecisas descripciones del resto de obras lexicográficas posteriores al Diccionario de autoridades, a excepción del de Terreros (1786) -de quien hablaremos a continuación-, parece ser que todos los lexicógrafos desconocían el origen y la forma de una catana. Así, desde Salvá (1846) hasta Zerolo (1895), las obras consultadas lo describen como «especie de alfanje chino». Alemany y Bolufer (1917), retoma el sema de 'indios' - incluido en el Diccionario de autoridades - para incluirlo en su definición y también amplía el concepto de 'chino': «especie de alfanje que usan los indios y otros pueblos orientales». Descripción que perduró hasta la vigésimoprimera edición del DRAE (1992). Únicamente Terreros y Pando da muestras explícitas de haber visto una catana:

dicen que es una especie de alfanje indiano, V. pero la catana que he visto en el curiosisimo gabinete del señor Conde de Saceda, en nada parece alfanje, pues es un palo ancho, labrado, sin corte, y con dos extremidades mas gruesas, y anchas que el resto $(1786: 380,2)$.

Este lexicógrafo se mostró prudente y no quiso contrariar a la Academia, de ahí el uso de dicen con la adversativa pero. Por eso, siguiendo la estela del Diccionario de autoridades, incluyó la forma masculina del término, si bien, precisando que se utilizaba «mas comunmente catana».

Para el origen de la forma singular y masculina, catán, podemos establecer varias teorías: por un lado, existe la posibilidad de que la grafía de Argensola o del impresor de su obra, se produjera a causa de una errata (se cambió la $a$ por la $e$ ). Por otro lado, pudo deberse a la interferencia de un italianismo homógrafo y homófono, catán, supuestamente presente en el español de la época y que venía siendo utilizado con el significado de 'infanzón'. Italianismo del que, por otro lado, ningún lexicógrafo ofrece el étimo y cuyo primer registro escrito data, según el CORDE, de 1491, en las Siete Partidas de Alfonso $X$ : «son llamados catanes \& vasuasores». El Diccionario de autoridades lo recoge a través de Covarrubias (1611: 1006: 2), y los dos, el Diccionario de autoridades y Covarrubias, hacen referencia al ejemplo del CORDE, donde viene empleado únicamente en plural: catanes. Encontramos otro ejemplo de catanes en el CORDE, que sigue la estela del originario: «que en otras partes se llaman catanes y valvasores», empleado por Mateo Luján de Saavedra (1602) en la Segunda parte de la vida del pícaro Guzman de Alfarache. En el diccionario histórico de la Academia $(1936: 864,2)$ se indica que, para su uso como sinónimo de 'infanzón', se emplea la forma paroxítona, catan, mientras que catán, aguda, se reserva para el significado de 'catana'. Sin embargo, tampoco hemos hallado ejemplos de significante singular, catan o catán, en los corpus del español con el significado de 'infanzón'. Por último, podemos formular a priori una hipótesis más convincente si tenemos en cuenta la información etimológica aportada por dos obras lexicográficas inglesas: según el $M W C D$, la voz inglesa catan o cattan procede de la española catán. El $O E D$ incluye también la variante cattan, y ofrece dos muestras: una en plural, Cattans (de 1613), y otra en singular, cattan (de 1615). Estos datos demuestran que en inglés de comienzos del siglo XVII existió una forma, catan o cattan $^{27}$, pero ¿hasta qué punto

${ }^{27}$ Debido a la carencia de género en la morfología de la lengua inglesa, desconocemos si se trataba de un sustantivo masculino o femenino. 
debemos confiar en los descriptores etimológicos de estos diccionarios? En los corpus españoles no hemos encontrado una sola muestra de masculino singular, catán, por lo que resulta evidente que tanto el $M W C D$, como el $O E D$, han obtenido su descriptor etimológico de la forma masculina singular presente en las obras lexicográficas españolas. Parece imposible, pues, sostener que en lengua española existiera una forma masculina singular, catán; no obstante, de haber contado con este significante, habría sido usado en rarísimas ocasiones durante un corto período de tiempo.

\section{CONCLUSIONES}

En definitiva, a menos que se encuentren ejemplos que demuestren que la voz podía ser utilizada también en masculino singular, con la pérdida de la vocal final /-a/ del étimo - fenómeno extraño en la historia de la lengua española ${ }^{28}$ - , lo cierto es que todo apunta a que en la redacción del Diccionario de autoridades, se creyó que el sustantivo plural empleado por Argensola, catanes, aun estando definido por el adjetivo femenino diuersas, procedía de una forma masculina y singular: catán. Y así se perpetuó en obras lexicográficas españolas hasta nuestros días, a pesar de que, según las muestras de los corpus, los hispanohablantes únicamente prefirieran la forma femenina, catana, que han venido utilizando a lo largo de casi cuatro siglos, comportándose de manera consecuente con el étimo japonés del que procede.

\section{REFERENCIAS BIBLIOGRÁFICAS}

Libros, manuales y artículos

CABEZAS, Antonio (1994): El siglo ibérico del Japón. La presencia hispano-portuguesa en Japón (1543-1643), Valladolid, Universidad de Valladolid.

CANo Aguilar, Rafael (2002 [1988]): El español a través de los tiempos, Madrid, Arco/Libros.

CiD LuCAS, Fernando, coord., et alii (2011): Japón y la Península Ibérica. Cinco siglos de encuentros, Gijón, Satori.

De Argensola, Bartolomé Leonardo (1609): Conquista de las islas Malucas [en línea]: $<$ http://books.google.pt/books/ucm?vid=UCM5323536934\&printsec=frontcover\& redir_esc $=\mathrm{y} \# \mathrm{v}=$ onepage \&q\&f$=$ false $>$ [Consulta: 07/06/2015].

DE MORGA, Antonio (1609): Sucesos de las islas Filipinas [en línea]: $<$ https://archive.org. stream/ahz9387.0001.001.umich.edu\#page/XXIV/mode/2up> [Consulta: 07/06/2015].

Fradejas Rueda, José Manuel (2000): Fonología histórica del español, Madrid, Visor Libros.

${ }^{28}$ Confróntense los trabajos de: Lapesa (2005 [1981 $\left.\left.{ }^{9}\right]\right)$, Cano Aguilar (2002 [1988]) o Fradejas (2000). Al perderse la vocal átona final, /-a/ — fenómeno extraño en lengua española, sobre todo si tenemos en cuenta su valor funcional como morfema de género-, inferimos que el significante español procedía del étimo japonés con patrón paroxítono, esto es, /katana/. Si bien, en una lengua tan próxima como la portuguesa, no solo lingüísticamente, sino también desde el punto de vista de contacto con el pueblo nipón, las primeras muestras de catana aparecen con patrón oxítono, / katana/, también presente, aunque menos, en lengua japonesa: catanà (1582) y catanás (1585), ambos recogidos por Dalgado (1919: 281), quien sostiene que era un patrón poco común, esto es, se preferían las formas paroxítonas en lengua portuguesa, sin pérdida de /-a/ final. 
GIL, Juan (1991): Hidalgos y samurais. España y Japón en los siglos XVI y XVII, Madrid, Alianza Editorial.

FraGO GRACIA, Juan Antonio (1997): «Japonesismos entre Acapulco y Sevilla: sobre biombo, catana y maque», Boletín de Filología de la Universidad de Chile, XXXVI, pp. 101-118.

LAPESA, Rafael (2005 [1981 $\left.\left.{ }^{9}\right]\right)$ : Historia de la lengua española, Madrid, Gredos.

Obras lexicográficas

AlmarZa, Nieves et al. (2012): Diccionario Clave: diccionario de uso del español actual [en línea] <http://clave.smdiccionarios.com/app.php> [Consulta: 16/04/2015].

Corominas, Joan y José Antonio PASCUAL (2012 [1991-1997]): Diccionario crítico etimológico castellano e hispánico, Madrid, Gredos (CD-ROM).

DALGADO, Sebastião Rodolfo (1919-1921): Glossário luso-asiático, Coímbra, Imprensa da Universidade.

Devoto, Giacomo y Gian Carlo Oli (2012): Il Devoto-Oli: vocabolario della lingua italiana 2013, Firenze, Le Monnier, 2012 (CD-ROM).

HouAiss, Antônio, dir. (2001): Dicionário Houaiss da Língua Portuguesa, Río de Janeiro, Editora Objectiva.

Lahuerta Galán, Javier, coord. (2003): Diccionario de uso del español de América y España, Barcelona, Vox (CD-ROM).

MatsumurA, Akira, dir. (2006): Daiyirín, Toquio, Sanseido Books [en línea] $<$ www.kotobank.jp> [Consulta: 16/04/2015].

MERriAM-WeBSTER (2003): Merriam-Webster's Collegiate Dictionary, Springfield, MerriamWebster (CD-ROM).

Moliner, María (2008 [1966-1967]): Diccionario de uso del español, Madrid, Gredos (CD-ROM).

Real ACAdemia Española (2001): Diccionario de la Real Academia Española, Madrid, Espasa-Calpe (CD-ROM).

Real ACAdemia Española (2014): Diccionario de la Real Academia Española, Madrid, Espasa Libros [en línea] <http://www.rae.es/> [Consulta: 16/04/2015].

SÁNCHEZ, Aquilino (2006 [2001]): Gran diccionario de uso del español actual, Madrid, SGEL (CD-ROM).

SeCo, Manuel, Olimpia de ANDRÉs y Gabino RAMOs (1999): Diccionario del español actual, Madrid, Aguilar.

SIMPSON, John, dir. (2009): Oxford English Dictionary, Oxford, Oxford University Press (CD-ROM, segunda edición 4.0).

VV.AA. (2016): Dicionário Priberam da Língua Portuguesa [en línea] $<$ http://www.priberam.pt/dlpo/> [Consulta: 16/04/2015].

ZINGARELLI, Nicola (2014): lo Zingarelli 2015. Vocabolario della lingua italiana [en línea] $<$ http://dizionari.zanichellipro.it/> [Consulta: 16/04/2015].

ANEXO I: LISTADO DE ABREVIACIONES EMPLEADAS

\begin{tabular}{|l|l|}
\hline CORDE : ........................................... Corpus Diacrónico del Español \\
\hline CORPES XXI .................................... Corpus del Español del Siglo XXI \\
\hline
\end{tabular}




\begin{tabular}{|c|c|}
\hline \multirow{2}{*}{\multicolumn{2}{|c|}{$\begin{array}{l}\text { CREA } \ldots \ldots \ldots \ldots \ldots \ldots \ldots \ldots \ldots \ldots \ldots \\
D C E C H\end{array}$}} \\
\hline & \\
\hline DClave... & Diccionario Clave: Diccionario de uso del español actual \\
\hline \multicolumn{2}{|c|}{ DEA .............................................. Diccionario del español actual } \\
\hline \multicolumn{2}{|c|}{ DHLP ............................................... Dicionário Houaiss da Língua Portuguesa } \\
\hline \multicolumn{2}{|c|}{ DPLP ................................................. Dicionário Priberam da Língua Portuguesa } \\
\hline \multicolumn{2}{|c|}{ DOVLI ............................................. Il Devoto-Oli: Vocabolario della lingua italiana } \\
\hline \multicolumn{2}{|c|}{ DRAE ................................................... Diccionario de la Real Academia Española } \\
\hline \multicolumn{2}{|c|}{ 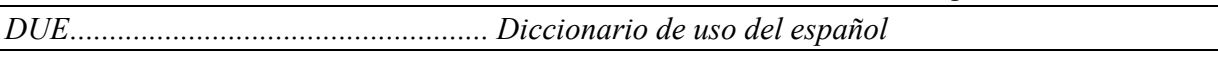 } \\
\hline \multicolumn{2}{|c|}{ DUEAE .............................................. Diccionario de uso del español de América y España } \\
\hline \multicolumn{2}{|c|}{ GDUEA ............................................ Gran diccionario de uso del español actual } \\
\hline \multicolumn{2}{|c|}{ MWCD.............................................. Merriam-Webster's Collegiate Dictionary } \\
\hline \multicolumn{2}{|c|}{ OED ........................................... Oxford English Dictionary } \\
\hline Zingarell & lo Zingarelli 2015. Vocabolario della lingua italiana \\
\hline
\end{tabular}

ANEXO II: INFORMACIÓN LEXICOGRÁFICA DEL NUEVO TESORO LEXICOGRÁFICO DE LA LENGUA ESPAÑOLA

\begin{tabular}{|c|c|c|}
\hline Diccionario & catán (masculino) & catana (femenino) \\
\hline 1706 STEVENS & - & Catana, a broad Sword. \\
\hline $\begin{array}{l}1729 \text { ACADEMIA } \\
\text { AUTORIDADES } \\
\text { (C) }\end{array}$ & $\begin{array}{l}\text { CATAN. s.m. Una espécie de } \\
\text { alfange que usan los Indios, que } \\
\text { en el Japón le llaman Catána. Lat. } \\
\text { Acinacis, is. ARGENS. Ma- } \\
\text { luc.lib.6.fol.200. Los quales } \\
\text { dieron sus muestras y reséñas con } \\
\text { picas y catánes. } \\
\text { CATAN. Lo mismo que Infan- } \\
\text { zón. Es voz Italiana; aunque } \\
\text { Covarr. dice puede venir del } \\
\text { Griego Katanemo, que significa } \\
\text { Distribuir ò dar. PAR- } \\
\text { TID.2.tit.x.l.x.3. Catánes è Val- } \\
\text { vasóres son aquellos fijosdalgo } \\
\text { en Italia, à que dicen en España } \\
\text { Infanzónes. }\end{array}$ & - \\
\hline $\begin{array}{l}1786 \text { TERREROS } \\
\text { y PANDO (A-F) }\end{array}$ & $\begin{array}{l}\text { CATÁN, voz Italiana, lo mismo } \\
\text { que infanzón, V. y el Dicc. De la } \\
\text { Acad. } \\
\text { CATÁN, y mas comunmente } \\
\text { catana, dicen que es una especie } \\
\text { de alfanje indiano, V. pero la } \\
\text { catana que he visto en el curiosi- } \\
\text { simo gabinete del señor Conde de } \\
\text { Saceda, en nada parece alfanje, } \\
\text { pues es un palo ancho, labrado, sin } \\
\text { corte, y con dos extremidades mas } \\
\text { gruesas, y anchas que el resto. }\end{array}$ & - \\
\hline $\begin{array}{l}1803 \text { ACADEMIA } \\
\text { SUPLEMENTO }\end{array}$ & 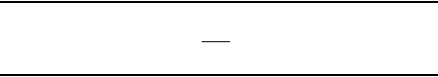 & $\begin{array}{l}\text { CATALINA. s. f. Especie de papagayo menor } \\
\text { que los comunes, y que tiene media cabeza }\end{array}$ \\
\hline
\end{tabular}




\begin{tabular}{|c|c|c|}
\hline & & $\begin{array}{l}\text { colorada. Psittaci genus. } \\
\text { CATANA, ó CATANLA. s. f. fam. nombre } \\
\text { propio de muger. Lo mismo que CATALINA. }\end{array}$ \\
\hline 1846 SALVÁ & $\begin{array}{l}\text { CATAN. m. Especie de alfanje } \\
\text { chino. } \| \text { ant. INFANZON: es voz } \\
\text { puramente italiana. }\end{array}$ & en \\
\hline $\begin{array}{l}1852 \text { CASTRO y } \\
\text { ROSSI (A- } \\
\text { COSTRA) }\end{array}$ & 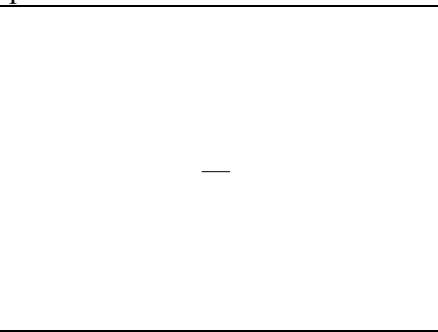 & $\begin{array}{l}\text { CATANA. s. f. Nombre de mujer. Lo mismo } \\
\text { que Catalina. } \\
\text { Ya parió Catana, ó gracias à Dios que Catana } \\
\text { parió, ó ya parió Catana y parió macho. } \\
\text { Andalucía y algunas partes de América y otras } \\
\text { de España. Manera de expresar la ejecución de } \\
\text { una cosa muy anunciada ó esperada. } \\
\text { CATANLA. s. f. Nombre de mujer. Lo mismo } \\
\text { que Catalina. }\end{array}$ \\
\hline $\begin{array}{l}1853 \text { DOMÍN- } \\
\text { GUEZ }\end{array}$ & - & $\begin{array}{l}\text { Catana ó Catina, Geog. C. de Sicilia, con } \\
80,000 \text { hab. Los temblores de tierra y las erup- } \\
\text { ciones del Etna la han arruinado varias veces; } \\
\text { en } 1669 \text { perecieron mas de } 18,000 \text { de sus hab. } \\
\text { Catanla, fam. V. CATALINA. }\end{array}$ \\
\hline $\begin{array}{l}1853 \text { DOMÍN- } \\
\text { GUEZ SUPLE- } \\
\text { MENTO }\end{array}$ & $\begin{array}{l}\text { CATAN, llamaron en Italia al } \\
\text { infanzon. }\end{array}$ & $\begin{array}{l}\text { CATANA, s. f. Ant. Arma que usaron los } \\
\text { antiguos semejante al alfange. Y dice Terreros } \\
\text { que en casa del conde de Saceda vió una cata- } \\
\text { na ó palo ancho, labrado, sin corte, y mas } \\
\text { grueso en las estremidades que en el centro. }\end{array}$ \\
\hline $\begin{array}{c}1853 \text { GASPAR y } \\
\text { ROIG (A-F) }\end{array}$ & $\begin{array}{l}\text { CATAN: s. m.: especie de alfanje } \\
\text { chino. -ant.: infanzon; es voz } \\
\text { puramente Italiana. }\end{array}$ & CATANA: s. f.: catan, en su primera acepcion. \\
\hline $\begin{array}{l}1869 \text { DOMÍN- } \\
\text { GUEZ SUPLE- } \\
\text { MENTO }\end{array}$ & $\begin{array}{l}\text { CATAN, s. m. Llamaron asi en } \\
\text { Italia al infanzon. \| Especie de } \\
\text { alfange chino. }\end{array}$ & $\begin{array}{l}\text { CATANA, s. f. Ant. Arma que usaron los } \\
\text { antiguos semejante al alfange, y dice Terreros } \\
\text { que en casa del conde de Saceda vió una cata- } \\
\text { na ó palo ancho, labrado, sin corte, y mas } \\
\text { grueso en las estremidades que en el centro. }\end{array}$ \\
\hline $\begin{array}{l}1879 \text { SALVÁ } \\
\text { SUPLEMENTO }\end{array}$ & - & $\begin{array}{l}\text { CATANA ó CATINA. Geog. C. de Sicilia, con } \\
80,000 \text { hab. Los temblores de tierra y las erup- } \\
\text { ciones del Etna la han arruinado varias veces. }\end{array}$ \\
\hline 1895 ZEROLO & $\begin{array}{l}\text { CATÁN. m. } 1 \text {. Especie de alfanje } \\
\text { chino. - } 2 \text {. ant. Infanzón: es voz } \\
\text { puramente italiana. }\end{array}$ & $\begin{array}{l}\text { CATANA. f. Amer. Ch. despect. Sable, y en } \\
\text { particular el de los guardias civiles y serenos } \\
\text { (Rou) } \\
\text { CATANA. Geogr. Cap. De la prov. Del mis- } \\
\text { mo nombre (Sicilia), en la costa oriental, al pie } \\
\text { del Etna. La ciudad tiene } 90,000 \text { hab. y la } \\
\text { prov. } 500,000 \text { con } 5,102 \mathrm{~km} \text {. cuadrados. }\end{array}$ \\
\hline $\begin{array}{l}1917 \text { ALEMANY } \\
\text { y BOLUFER }\end{array}$ & $\begin{array}{l}\text { CATAN. (Del ár. jatán, sable) m. } \\
\text { Especie de alfanje que usan los } \\
\text { indios y otros pueblos orientales. }\end{array}$ & $\begin{array}{l}\text { CATANA. f. Catán. Amér. En Chile y Repú- } \\
\text { blica Argentina, sable, especialmente el largo y } \\
\text { viejo y que usan los polizontes; chafarote. } \\
\text { Amér. En Cuba, cosa pesada, tosca, deforme. } \\
\text { Amér. En Venezuela, loro verde y azul. }\end{array}$ \\
\hline $\begin{array}{l}1918 \text { RODRÍ- } \\
\text { GUEZ NAVAS }\end{array}$ & $\begin{array}{l}\text { catan, m. Alfange que usan in- } \\
\text { dios y otros orientales. Del ár. } \\
\text { jatan, sable. }\end{array}$ & $\begin{array}{l}\text { Catana, f. Catán. Chafarote o sable de policía } \\
\text { en la Argentina y Chile. Cuba. Cosa tosca, } \\
\text { pesada, deforme. Venezuela. Loro azul y verde. }\end{array}$ \\
\hline
\end{tabular}




\begin{tabular}{|c|c|c|}
\hline $\begin{array}{l}1925 \text { ACADEMIA } \\
\text { USUAL }\end{array}$ & $\begin{array}{l}\text { CATÁN. (Del ár. jatán, sable). } \\
\text { m. Especie de alfanje que usaban } \\
\text { los indios y otros pueblos del } \\
\text { Oriente. }\end{array}$ & $\begin{array}{l}\text { CATANA. f. Catán. 2. Argent. y Chile. Sable, } \\
\text { en especial el largo y viejo, y el que usan los } \\
\text { policías. Es voz despectiva. 3. Cuba. Cosa } \\
\text { pesada, tosca, deforme. 4. Venez. Loro verde y } \\
\text { azul. Hay otras variedades. }\end{array}$ \\
\hline $\begin{array}{l}1927 \text { ACADEMIA } \\
\text { MANUAL }\end{array}$ & $\begin{array}{l}\text { CATÁN. m. Especie de alfanje } \\
\text { que usaban los indios y otros } \\
\text { pueblos del Oriente. }\end{array}$ & $\begin{array}{l}\text { CATANA. f. Catán. Argent. y Chile. Sable, en } \\
\text { especial el largo y viejo, y el que usan los } \\
\text { policías. Es voz despectiva. Cuba. Cosa pesa- } \\
\text { da, tosca, deforme. Venez. Loro verde y azul. } \\
\text { Hay otras variedades. }\end{array}$ \\
\hline $\begin{array}{l}1936 \text { ACADEMIA } \\
\text { HISTORICO } \\
\text { (B-Cevilla) }\end{array}$ & $\begin{array}{l}\text { CATAN. m. ant. Infanzón. } \\
\text { CATÁN. (Del ár. jatán, sable). } \\
\text { m. Especie de alfanje que usaban } \\
\text { los indios y otros pueblos del } \\
\text { Oriente. }\end{array}$ & $\begin{array}{l}\text { CATANA. f. Catán, } 2^{\circ} \text { art. 2. Argent. y Chile. } \\
\text { Sable, en especial el largo y viejo. 3. Cuba. } \\
\text { Cosa deforme. } \\
\text { CATANA. (De Catalina). f. Venez. Loro verde } \\
\text { y azul. Hay otras variedades. } \\
\text { CATANA. f. Bilbao. Borrachera. } \\
\text { CATANA. f. Amér. Barca o esquife. }\end{array}$ \\
\hline $\begin{array}{l}1936 \text { ACADEMIA } \\
\text { USUAL }\end{array}$ & $\begin{array}{l}\text { CATÁN. (Del ár. jatán, sable). } \\
\text { m. Especie de alfanje que usaban } \\
\text { los indios y otros pueblos del } \\
\text { Oriente. }\end{array}$ & $\begin{array}{l}\text { Catana. f. Catán. I| 2. Argent. y Chile. Sable, en } \\
\text { especial el largo y viejo, y el que usan los } \\
\text { policías. Es voz despectiva. \| 3. Cuba. Cosa } \\
\text { pesada, tosca, deforme. \| 3. Venez. Loro verde } \\
\text { y azul. Hay otras variedades. }\end{array}$ \\
\hline $\begin{array}{l}1939 \text { ACADEMIA } \\
\text { USUAL }\end{array}$ & $\begin{array}{l}\text { CATÁN. (Del ár. jatán, sable). } \\
\text { m. Especie de alfanje que usaban } \\
\text { los indios y otros pueblos del } \\
\text { Oriente. }\end{array}$ & $\begin{array}{l}\text { Catana. f. Catán. \| 2. Argent. y Chile. Sable, en } \\
\text { especial el largo y viejo, y el que usan los } \\
\text { policías. Es voz despectiva. \| 3. Cuba. Cosa } \\
\text { pesada, tosca, deforme. } \| 3 \text {. Venez. Loro verde } \\
\text { y azul. Hay otras variedades. }\end{array}$ \\
\hline $\begin{array}{l}1947 \text { ACADEMIA } \\
\text { USUAL }\end{array}$ & $\begin{array}{l}\text { CATÁN. (Del ár. jatán, sable). } \\
\text { m. Especie de alfanje que usaban } \\
\text { los indios y otros pueblos del } \\
\text { Oriente. }\end{array}$ & $\begin{array}{l}\text { Catana. f. Catán. \| 2. Argent. y Chile. Sable, en } \\
\text { especial el largo y viejo, y el que usan los } \\
\text { policías. Es voz despectiva. \| 3. Cuba. Cosa } \\
\text { pesada, tosca, deforme. } \| 3 \text {. Venez. Loro verde } \\
\text { y azul. Hay otras variedades. }\end{array}$ \\
\hline $\begin{array}{l}1950 \text { ACADEMIA } \\
\text { MANUAL }\end{array}$ & $\begin{array}{l}\text { CATÁN. m. Especie de alfanje } \\
\text { que usaban los indios y otros } \\
\text { pueblos del Oriente. }\end{array}$ & $\begin{array}{l}\text { CATANA. f. Catán. \| Argent. y Chile. Sable, } \\
\text { en especial el largo y viejo, y el que usan los } \\
\text { policías. Es voz despectiva. \| Cuba. Cosa } \\
\text { pesada, tosca, deforme. \|Venez. Loro verde y } \\
\text { azul. Hay otras variedades. }\end{array}$ \\
\hline $\begin{array}{l}1956 \text { ACADEMIA } \\
\text { USUAL }\end{array}$ & $\begin{array}{l}\text { Catán. (Del ár. qat } t^{\star} \bar{a} \text { ', cortante, } \\
\text { dicho de una espada.) m. Especie } \\
\text { de alfanje que usaban los indios y } \\
\text { otros pueblos del Oriente. }\end{array}$ & $\begin{array}{l}\text { Catana. f. Catán. \| 2. Argent. y Chile. Sable, en } \\
\text { especial el largo y viejo, y el que usan los } \\
\text { policías. Es voz despectiva. \| 3. Cuba. Cosa } \\
\text { pesada, tosca, deforme. \| 3. Venez. Loro verde } \\
\text { y azul. Hay otras variedades. }\end{array}$ \\
\hline $\begin{array}{l}1970 \text { ACADEMIA } \\
\text { USUAL }\end{array}$ & $\begin{array}{l}\text { Catán. (Del ár. qat } t^{\star} \bar{a} \text {, cortante, } \\
\text { dicho de una espada.) m. Especie } \\
\text { de alfanje que usaban los indios y } \\
\text { otros pueblos del Oriente. }\end{array}$ & $\begin{array}{l}\text { catana }{ }^{1} \text {. f. Catán. } \| 2 \text {. Argent. y Chile. Sable, en } \\
\text { especial el largo y viejo, y el cual usan los } \\
\text { policías. Es voz despectiva. \| 3. Cuba. Cosa } \\
\text { pesada, tosca, deforme. } \\
\text { catana }{ }^{2} \text {. (De Catalina) f. Venez. Loro verde y } \\
\text { azul. Hay otras variedades. }\end{array}$ \\
\hline 1983 ACADEMIA & catán. m. Especie de alfanje que & catana. f. catán. $\|$ Argent. y Chile. Sable, en \\
\hline MANUAL TOMO & usaban los indios y otros pueblos & especial el largo y viejo, y el que usan los \\
\hline II (Capacho-Divo) & & policías. Es voz despectiva. \| Cuba. Cosa \\
\hline
\end{tabular}




\begin{tabular}{|c|c|c|}
\hline & & $\begin{array}{l}\text { pesada, tosca, deforme. II Venez. Loro verde y } \\
\text { azul. Hay otras variedades. }\end{array}$ \\
\hline $\begin{array}{l}1984 \text { ACADEMIA } \\
\text { USUAL }\end{array}$ & $\begin{array}{l}\text { catán. (Del ár. } q a t^{\star} \bar{a}^{\prime} \text {, cortante, } \\
\text { dicho de una espada.) m. Especie } \\
\text { de alfanje que usaban los indios y } \\
\text { otros pueblos del Oriente. }\end{array}$ & 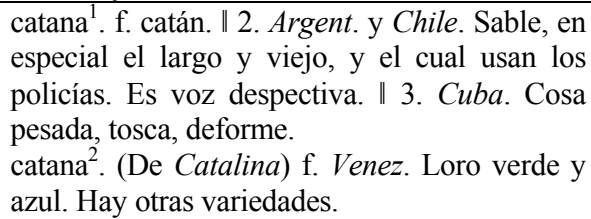 \\
\hline $\begin{array}{l}1989 \text { ACADEMIA } \\
\text { MANUAL }\end{array}$ & $\begin{array}{l}\text { catán. m. Especie de alfanje que } \\
\text { usaban los indios y otros pueblos } \\
\text { del Oriente. }\end{array}$ & $\begin{array}{l}\text { catana. f. catán. Argent. y Chile. Sable, en } \\
\text { especial el largo y viejo, y el que usan los } \\
\text { policías. Es voz despectiva. \| Cuba. Cosa } \\
\text { pesada, tosca, deforme. \|Venez. Loro verde y } \\
\text { azul. Hay otras variedades. }\end{array}$ \\
\hline $\begin{array}{l}1992 \text { ACADEMIA } \\
\text { USUAL }\end{array}$ & $\begin{array}{l}\text { catán. (Del ár. qat' } \bar{a} \text {, cortante, } \\
\text { dicho de una espada.) m. Especie } \\
\text { de alfanje que usaban los indios y } \\
\text { otros pueblos del Oriente. }\end{array}$ & $\begin{array}{l}\text { catana }{ }^{1} \text {. f. catán. \| 2. despect. Chile. Sable, en } \\
\text { especial el largo y viejo, que usaban los poli- } \\
\text { cías. } \| \text { 3. Cuba. Cosa pesada, tosca, deforme. } \\
\text { catana }{ }^{2} \text {. (De Catalina, n. p.; cf. caley) f. Venez. } \\
\text { Loro verde y azul. Hay otras variedades. }\end{array}$ \\
\hline $\begin{array}{l}2001 \text { ACADEMIA } \\
\text { USUAL }\end{array}$ & $\begin{array}{l}\text { catán. } 1 . \text { m. catana (\| especie de } \\
\text { alfanje). }\end{array}$ & $\begin{array}{l}\text { catana }{ }^{1} \text {. Del jap. katana, espada). 1. f. Especie } \\
\text { de alfanje que usaban los indios y otros pue- } \\
\text { blos del Oriente. } 2 \text {. f. despect. Chile. Sable, en } \\
\text { especial el largo y viejo, que usaban los poli- } \\
\text { cías. 3. f. Cuba. Automóvil o aparato mecánico } \\
\text { viejo y destartalado. } \\
\text { catana }{ }^{2} \text {. (De Catalina, n. p). } \\
\text { 1. f. Ven. Loro verde y azul. Hay otras varie- } \\
\text { dades. }\end{array}$ \\
\hline $\begin{array}{l}2014 \text { ACADEMIA } \\
\text { USUAL }\end{array}$ & $\begin{array}{l}\text { catán. } 1 . \text { m. catana (\| especie de } \\
\text { alfanje). }\end{array}$ & $\begin{array}{l}\text { catana }^{1} \text {. (Del jap. katana, espada). 1. f. Especie } \\
\text { de alfanje que usaban los indios y otros pue- } \\
\text { blos del Oriente. } 2 \text {. f. despect. Chile. Sable, en } \\
\text { especial el largo y viejo, que usaban los poli- } \\
\text { cías. 3. f. Cuba. Automóvil o aparato mecánico } \\
\text { viejo y destartalado. } \\
\text { catana }{ }^{2} \text {. (De Catalina, n. p). } \\
\text { 1. f. Ven. Loro verde y azul. Hay otras varie- } \\
\text { dades. }\end{array}$ \\
\hline
\end{tabular}

